\title{
Strategy for tuning the average charge state of metal ions incident atthe growing film during HIPIMS deposition
}

\author{
Grzegorz Greczynski, Ivan Petrov, Joseph E Greene and Lars Hultman
}

\section{Linköping University Post Print}

\section{Tweet}

N.B.: When citing this work, cite the original article.

Original Publication:

Grzegorz Greczynski, Ivan Petrov, Joseph E Greene and Lars Hultman, Strategy for tuning the average charge state of metal ions incident atthe growing film during HIPIMS deposition, 2015, Vacuum, (116), 36-41.

http://dx.doi.org/10.1016/j.vacuum.2015.02.027

Copyright: Elsevier http://www.elsevier.com/

Postprint available at: Linköping University Electronic Press

http://urn.kb.se/resolve?urn=urn:nbn:se:liu:diva-118602 


\title{
Strategy for tuning the average charge state of metal ions incident at the growing film during HIPIMS deposition
}

\author{
G. Greczynski, ${ }^{1}$ I. Petrov, ${ }^{1,2}$ J.E. Greene, ${ }^{1,2,3}$ and L. Hultman ${ }^{1}$ \\ ${ }^{1}$ Department of Physics (IFM), Linköping University, SE-581 83 Linköping, Sweden \\ ${ }^{2}$ Materials Science Department and Frederick Seitz Materials Research Laboratory, University of \\ Illinois, Urbana, Illinois 61801 \\ ${ }^{3}$ Department of Physics, University of Illinois, Urbana, Illinois 61801, USA
}

\begin{abstract}
Energy- and time-dependent mass spectrometry is used to determine the relative number density of singly- and multiply-charged metal-ion fluxes incident at the substrate during highpower pulsed magnetron sputtering (HIPIMS) as a function of the average noble-gas ionization potential. $\mathrm{Ti}$ is selected as the sputtering target since the microstructure, phase composition, properties, and stress-state of Ti-based ceramic thin films grown by HIPIMS are known to be strongly dependent on the charge state of $\operatorname{Ti}^{n+}(n=1,2, \ldots)$ ions incident at the film growth surface. We find that the flux of $\mathrm{Ti}^{n+}$ with $n>2$ is insignificant; thus, we measure the $\mathrm{Ti}^{2+} / \mathrm{Ti}^{+}$integrated flux ratio $J_{T i^{2+}} / J_{T i^{+}}$at the substrate position as a function of the choice of noble gas -- $\mathrm{Ne}, \mathrm{Ar}, \mathrm{Kr}$, $\mathrm{Xe}$, as well as $\mathrm{Ne} / \mathrm{Ar}, \mathrm{Kr} / \mathrm{Ar}$, and $\mathrm{Xe} / \mathrm{Ar}$ mixtures -- supporting the plasma. We demonstrate that by changing noble-gas mixtures, $J_{T i^{2+}}$ varies by more than two orders of magnitude with only a small change in $J_{T i^{+}}$. This allows the ratio $J_{T i^{2+}} / J_{T i^{+}}$to be continuously tuned from less than 0.01 with $\mathrm{Xe}$, which has a low first-ionization potential $I P^{1}$, to 0.62 with $\mathrm{Ne}$ which has a high $I P^{1}$. The value for $\mathrm{Xe}, I P_{X e}^{1}=12.16 \mathrm{eV}$, is larger than the first ionization potential of $\mathrm{Ti}, I P_{T i}^{1}=6.85 \mathrm{eV}$, but less than the second Ti ionization potential, $I P_{T i}^{2}=13.62 \mathrm{eV}$. For Ne, however, $I P_{N e}^{1}=21.63 \mathrm{eV}$ is greater than both $I P_{T i}^{1}$ and $I P_{T i}^{2}$. Therefore, the high-energy tail of the plasma-electron energy distribution can be systematically adjusted, allowing $J_{T i^{2+}} / J_{T i^{+}}$to be controllably varied over a very wide range.
\end{abstract}

corresponding author: grzgr@ifm.liu.se; phone: +4613281213

Keywords: HIPIMS; HPPMS; ionized PVD; ion charge state; 


\section{Introduction}

Low-energy metal-ion irradiation of growing films during high-power pulsed magnetron sputter deposition (HIPIMS), ${ }^{1}$ with the substrate bias synchronized to the metal-ion-rich portion of the pulses, ${ }^{2,3}$ has recently been shown to provide microstructure densification and surface smoothening, without introducing the large increases in film compressive stress reported for noblegas ion irradiation during dc magnetron sputter deposition, ${ }^{4,5}$ provided that the metal-ion flux incident at the substrate is primarily singly-ionized. In contrast, multiply-charged metal-ion irradiation, even at floating potential, can result in films with high defect densities and high compressive stresses. ${ }^{2,3}$ Synchronized-bias HIPIMS $\mathrm{CrN}$ films deposited in mixed $\mathrm{Ar} / \mathrm{N}_{2}$ atmospheres with high substrate bias and a significant doubly-charged metal-ion component, $J_{C r^{2+}} / J_{C r^{+}}=0.23$, exhibit compressive stresses up to $-9.6 \mathrm{GPa}^{6,7}$

Film stress increases rapidly as the average metal-ion momentum per deposited atom $\left\langle p_{d}\right\rangle$ transferred to the film surface exceeds a critical value $\left\langle p_{d}\right\rangle^{*}$, which depends upon the choice of materials system and the ion flux incident at the growth surface. ${ }^{8}\left\langle p_{d}\right\rangle$ is defined as $\sqrt{2 m_{i} n_{i} e\left(V_{s}-V_{p l}\right)} \times J_{i o n} / J_{M e}$ in which $V_{p l}$ is the plasma potential, $m_{i}$ and $n_{i}$ are the ion mass and average charge state, and $J_{i o n} / J_{M e}$ is the ratio of incident metal-ion to total metal (ion plus atom) fluxes. During deposition by HIPIMS, and hybrid techniques incorporating HIPIMS and dc magnetron co-sputtering (HIPIMS/DCMS), ${ }^{2}\left\langle p_{d}\right\rangle^{*}$ for growth of films with high-mass metal constituents can easily be exceeded, even with no applied substrate bias $V_{s .}\left\langle p_{d}\right\rangle$ is further increased for higher-mass metals with second-ionization potentials $I P_{M e}^{2}$ lower than the first-ionization potential $I P_{g}^{1}$ of the sputtering gas, leading to the production of high fluxes of multiply-charged ( $n$ >1) metal ions. ${ }^{8}$

It is therefore of interest to systematically vary the noble-gas composition, and hence the gas ionization potential, during HIPIMS and explore the effect of the average $I P_{g}^{1}$ value on the 
metal-ion charge state distribution. We use $\mathrm{Ti}$ as a model target material and employ energy- and time-dependent mass spectrometry to measure the integrated fluxes $J_{T i^{n+}}(n=1,2, \ldots)$ of metal ions incident at the substrate during Ti HIPIMS in $\mathrm{Ar}, \mathrm{Ne}, \mathrm{Kr}, \mathrm{Xe}$, and mixed noble-gas plasmas. The average value of $I P_{g}^{1}$ can be tuned by systematically adjusting mixed noble-gas compositions, thereby continuously altering the high-energy tail of the plasma-electron-energy distribution and controllably varying $J_{T i^{2+}} / J_{T i^{+}}$over a wide range from 0.01 to 0.62 .

\section{Experimental procedure}

HIPIMS experiments are carried out in a CemeCon CC800/9 magnetron sputtering system. A cast rectangular $8.8 \times 50 \mathrm{~cm}^{2}$ Ti target $(99.99 \%$ pure) is operated in HIPIMS mode with the average power $\mathcal{P}$ set to $1.0 \mathrm{~kW}$, a pulsing frequency of $100 \mathrm{~Hz}$ ( $2 \%$ duty cycle), and an energy per pulse (the product of target voltage and target current integrated over the entire pulse) of $E_{p}=10$ J. The total pressure of $\mathrm{Ne}, \mathrm{Ar}, \mathrm{Kr}, \mathrm{Xe}$, and Ar-based gas mixtures is maintained constant at $P=$ $0.4 \mathrm{~Pa}$ (3 mTorr). For sputtering with gas mixtures, the relative noble-gas flow rate $\chi_{g}=f_{g} /\left(f_{g}+f_{A r}\right)$, in which $f_{g}$ and $f_{A r}$ are mass flows, is varied from 0 to 1.

Time-dependent in-situ mass and energy spectroscopy analyses of ion fluxes incident at the substrate plane are performed using a Hiden Analytical EQP1000 instrument. The orifice of the mass spectrometer is placed at the substrate position, parallel to the target surface, and $18 \mathrm{~cm}$ from its center. The composition, charge state, and energy of ion fluxes incident at the substrate plane are determined as a function of $\chi_{g}$. Ion-energy distribution functions $I_{T i^{n+}}\left(E_{i}\right)$ (IEDFs) are recorded in HIPIMS mode for $\operatorname{Ti}^{n+}(n=1,2, \ldots)$ metal and gas ions during 50 consecutive $200-\mu \mathrm{s}$ pulses such that the total acquisition time per data point is $1 \mathrm{~ms}$. The ion energy is scanned in $1 \mathrm{eV}$ steps from $E_{i}=1 \mathrm{eV}$ to $E_{i}^{\prime}$, corresponding to the energy at which the $I_{T i^{n+}}$ flux intensity decreases 
to $\leq 1 \%$ of the maximum value $I_{T i^{n+}}^{\max }$. For $\mathrm{Ar}, \mathrm{Kr}$, and $\mathrm{Xe}, I_{T i^{+}}$becomes $\leq 0.01 \times I_{T i^{+}}^{\max }$ at $E_{i}^{\prime} \leq 30$ $\mathrm{eV}$, while $E_{i}^{\prime}$ increases to $>50 \mathrm{eV}$ in Ne discharges. For the case of lower-intensity $\mathrm{Ti}^{2+}$ ion fluxes (e.g., Ti sputtered in $\mathrm{Kr}$ and $\mathrm{Xe}$ ), $E_{i}^{\prime}$ is set by the detection limit of the mass spectrometer. Here, for consistency, we show IEDFs plotted as a function of $1 \leq E_{i} \leq 30 \mathrm{eV}$. Additional details associated with the IEDF measurements are given in Ref. 9.

\section{Results}

\section{III.A. Discharge characteristics}

HIPIMS target current density waveforms $j_{T}(t)$ acquired during sputtering of $\mathrm{Ti}$ in mixed noble gases -- Ne/Ar, $\mathrm{Kr} / \mathrm{Ar}$, and $\mathrm{Xe} / \mathrm{Ar}$-- with $\chi_{g}$ varied from 0 to 1 in each case, are shown in Figure 1. All pulse shapes are characterized by a steep rise during the first $30-50 \mu$ s, followed by a more gradual decrease during the next $80-100 \mu$ s due to depletion of the power-supply capacitor bank

For pure noble-gas discharges, $j_{T}(t)$ reaches a maximum at $\tau_{\max }=63,36,49$, and $57 \mu$ s into the pulse while sputtering with $\mathrm{Ne}, \mathrm{Ar}, \mathrm{Kr}$, and $\mathrm{Xe}$, respectively. Maximum $j_{T}(t)$ values $j_{\max }$ vary from $0.75 \mathrm{~A} / \mathrm{cm}^{2}$ for $\mathrm{Xe}$, to $0.80,1.05$, and $1.06 \mathrm{~A} / \mathrm{cm}^{2}$ for $\mathrm{Kr}, \mathrm{Ne}$, and Ar. The onset of the $j_{T}(t)$ pulse occurs, following a delay, at $\tau_{o n} \sim 2 \mu \mathrm{s}$, in $\mathrm{Ar}, \mathrm{Kr}$, and Xe discharges. With $\mathrm{Ne}, \tau_{o n}$ is $\sim 25 \mu \mathrm{s}$, more than an order of magnitude larger, due to a significantly lower electron-impact ionization cross-section $\sigma_{i}$ (see Table 1).

For HIPIMS sputtering in Ne/Ar mixtures, Fig. 1(a), $j_{\max }$ values are essentially independent of $\chi_{N e}$ at $1.04 \pm 0.02 \mathrm{~A} / \mathrm{cm}^{2}$. The time $\tau_{\max }$ into the pulse at which $j_{T}(t)$ reaches a maximum ranges from $63 \mu$ s with $\chi_{N e}=1$ to $41,39,38 \mu$ s with $\chi_{N e}=0.75,0.60,0.40$, and remains essentially constant at $36 \mu$ s for $0.25 \geq \chi_{N e} \geq 0$. The pulse onset time $\tau_{o n}$ decreases sharply upon addition of Ar, from 
$25 \mu$ s with $\chi_{N e}=1$, to $8,5,4,3,2.5$, and $2 \mu$ s with $\chi_{\text {Ne }}=0.75,0.60,0.40,0.25,0.1$, and 0 . The overall $j_{T}(t)$ peak shape does not vary significantly with $\chi_{\text {Ne }}$.

$j_{T}(t)$ waveforms for $\mathrm{Kr} / \mathrm{Ar}$ mixtures are plotted as a function of $\chi_{K r}$ in Fig. 1(b). Both pulse shapes and $j_{\max }$ values are strongly dependent on the $\mathrm{Kr}$ fraction. As $\chi_{K r}$ is decreased from 1 to $0.75,0.60,0.40,0.25$, and $0.10, j_{T}(t)$ maxima gradually increase from $0.80 \mathrm{~A} / \mathrm{cm}^{2}$ to $0.86,0.91$, $0.95,1.00$, and $1.03 \mathrm{~A} / \mathrm{cm}^{2}$ with a corresponding decrease in $\tau_{\max }$ from $49 \mu$ s for pure $\mathrm{Kr}$ to 45,43 , 40,38 , and $37 \mu \mathrm{s}$, respectively. The pulse onset time $\tau_{o n}$ is $\sim 2 \mu$ s for all $\chi_{K r}$ values.

$\mathrm{Xe} / \mathrm{Ar}$ waveform shapes as a function of $\chi_{X e}$, Figure 1(c), are similar to those for $\mathrm{Kr} / \mathrm{Ar}$ (Fig. 1(b)). $j_{\max }$ increases and $\tau_{\max }$ decreases with decreasing Xe fraction in the gas mixture. $j_{\max }$ is 0.75 $\mathrm{A} / \mathrm{cm}^{2}\left(\tau_{\max }=57 \mu \mathrm{s}\right)$ with $\chi_{X e}=1$ and increases to $0.82 \mathrm{~A} / \mathrm{cm}^{2}(52 \mu \mathrm{s}), 0.85(48), 0.90$ (45), 0.95 (42), and $0.98 \mathrm{~A} / \mathrm{cm}^{2}(40 \mu \mathrm{s})$ with $\chi_{X e}=0.75,0.60,0.40,0.25$, and 0.10 .

Figure 1 reveals a marked delay in the current pulse onset in pure $\mathrm{Ne}$ compared to $\mathrm{Ar}, \mathrm{Kr}$, $\mathrm{Xe}$, and noble-gas mixtures. This is primarily due to the fact that Ne has a much lower electronimpact ionization cross section $\sigma_{i}$ over the entire electron-energy range. ${ }^{10}$ Noble-gas $\sigma_{i}$ values exhibit, by definition, a threshold at $I P_{g}^{1}$ and the threshold is highest for $\mathrm{Ne}$ (see Table 1). At electron energies $E_{e}>I P_{g}^{1}, \sigma_{i} v s . E_{e}$ curves have broad maxima, which for Ne occurs at $\sim 160 \mathrm{eV}$ compared to $70-90 \mathrm{eV}$ for the heavier noble gases. In addition, $\sigma_{N e}$ is markedly lower, by $4 \times$ to $7 \times$, than corresponding values for $\mathrm{Ar}, \mathrm{Kr}$, and $\mathrm{Xe}$, which are grouped more closely together, for all electron energies. The small $\sigma_{N e}$ value makes it more difficult to strike a $\mathrm{Ne}$ discharge and thus increases $\tau_{o n}$ in Fig. 1, analogous to reported observations that the low-pressure limit for striking Ne magnetron discharges is $7 \times$ to $10 \times$ higher than for Ar, Kr, or Xe. ${ }^{11}$ Adding relatively small amounts of Ar to Ne decreases $\tau_{o n}$ as the lower ionization potential of Ar facilitates plasma ignition. 
III.B. Mass and energy analyses of ions incident at the substrate position

For all gas compositions, the only detected ionized sputter-ejected target atoms incident at the substrate plane are $\mathrm{Ti}^{+}$and $\mathrm{Ti}^{2+}$. Figs. 2(a) and 2(b) are $\mathrm{Ti}^{+}$and $\mathrm{Ti}^{2+}$ IEDFs for $\mathrm{Ne}, \mathrm{Ar}, \mathrm{Kr}$, and Xe HIPIMS plasmas. Ar, $\mathrm{Kr}$, and $\mathrm{Xe} \mathrm{Ti}^{+}$IEDFs exhibit similar shapes characterized by broad ionenergy distributions together with an $I_{T i^{+}}$peak at low energy, $~ 3-4 \mathrm{eV}$, corresponding to sputterejected species which are thermalized during transport in the gas phase and hence arrive late in the HIPIMS pulse. ${ }^{9}$ The integrated average incident ion energy $\left\langle E_{\mathrm{Ti}^{+}}\right\rangle$is $6.4 \mathrm{eV}$ for $\mathrm{Ar}, 6.8 \mathrm{eV}$ for $\mathrm{Kr}$, and $7.1 \mathrm{eV}$ for $\mathrm{Xe}$. $\mathrm{Ti}^{+}$IEDFs recorded while sputtering in $\mathrm{Ne}$ also have a broad energy distribution, but without the strong low-energy peak obtained with the other noble-gases. In addition, Ne-based $\mathrm{Ti}^{+}$IEDFs have a much more pronounced high-energy tail giving rise to a much higher average ion energy, $\left\langle E_{T i^{+}}\right\rangle=15.6 \mathrm{eV}$. The integrated area $J_{T i^{+}}$under the $I_{T i^{+}}\left(E_{i}\right)$ curves, $J_{T i^{+}}=$ $\int_{E_{i}=1}^{E_{i}=E_{i}^{\prime}} I_{T i^{+}}\left(E_{i}\right) d E_{i}$, corresponding to the total flux of $\mathrm{Ti}^{+}$ions with $E_{i} \leq E_{i}^{\prime}$, is $3.4 \times 10^{8} \mathrm{cps}$ for Ne; $J_{T i^{+}}$decreases with increasing noble-gas atomic number to $2.6 \times 10^{8}, 2.2 \times 10^{8}$, and $1.2 \times 10^{8} \mathrm{cps}$ for $\mathrm{Ar}, \mathrm{Kr}$, and $\mathrm{Xe}$. The maximum range in $J_{T i^{+}}$, from $\mathrm{Ne}$ to $\mathrm{Xe}$, is $2.8 \times$.

The $\mathrm{Ti}^{2+}$ IEDFs, Fig. 2(b), are much more sensitive than $\mathrm{Ti}^{+}$IEDFs to the choice of sputtering gas and $I_{\mathrm{Ti}^{2+}}\left(E_{i}\right)$ decreases more slowly with ion energy. Integrated areas under $\mathrm{Ne} \mathrm{Ti}^{2+} \mathrm{IEDFs}$ correspond to $J_{T i^{2+}}=2.1 \times 10^{8} \mathrm{cps} . J_{T i^{2+}}$ decreases dramatically with increasing atomic number of the sputtering gas to $4.5 \times 10^{7} \mathrm{cps}$ for $\mathrm{Ar}, 6.3 \times 10^{6} \mathrm{cps}$ for $\mathrm{Kr}$, and $1.2 \times 10^{6} \mathrm{cps}$ for $\mathrm{Xe}$. Thus, from $\mathrm{Ne}$ to $\mathrm{Xe}, J_{T i^{2+}}$ drops by a factor of $175 \times$, dramatically larger than the corresponding change in $J_{T i^{+}}$. The average $\mathrm{Ti}^{2+}$ ion energy $\left\langle E_{T i^{2+}}\right\rangle$ is also substantially higher for $\mathrm{Ne}, 25.0 \mathrm{eV}$, than for the other noble gases: $10.9 \mathrm{eV}$ for $\mathrm{Ar}, 12.3 \mathrm{eV}$ for $\mathrm{Kr}$, and $14.0 \mathrm{eV}$ for $\mathrm{Xe}$.

Fig. 3 is a plot of the doubly-to-singly charged Ti ion flux ratio, $J_{T i^{2+}} / J_{T i^{+}}$, as a function of the sputtering gas composition $\chi_{g}$. Of the four noble gases, $J_{T i^{2+}} / J_{T i^{+}}=0.62$ is highest for $\mathrm{Ne}$, 
which has the highest first ionization potential $I P_{N e}^{1}$ value, $21.63 \mathrm{eV}$ (see Table 1$) . J_{T i^{2+}} / J_{T i^{+}}$ decreases to 0.17 for Ar, to 0.03 for $\mathrm{Kr}$, and 0.01 for Xe which has the lowest first ionization potential, $I P_{X e}^{1}=12.16 \mathrm{eV}$. Thus, $J_{T i^{2+}} / J_{T i^{+}}$can be varied step-wise over a wide range from 0.01 to 0.62 simply by changing the sputtering gas. Controlled continuous tuning of $J_{T i^{2+}} / J_{T i^{+}}$is obtained through the use of gas mixtures, as illustrated in Figure 3. For Ne/Ar, $J_{T i^{2+}} / J_{T i^{+}}$ranges from 0.17 (pure Ar) to 0.62 (pure Ne); in $\mathrm{Kr} / \mathrm{Ar}$ mixtures, $0.03 \leq J_{T i^{2+}} / J_{T i^{+}} \leq 0.17$; and for Xe/Ar, $0.01 \leq J_{T i^{2+}} / J_{T i^{+}} \leq 0.17$

An alternative approach to varying the doubly-to-singly charged Ti ion flux ratio $J_{T i^{2+}} / J_{T i^{+}}$ is to vary the HIPIMS pulse parameters, which we explore here and compare with the above results for adjusting noble-gas mixtures. Since ionization of sputter-ejected species in HIPIMS discharges is predominantly due to electron impact, ${ }^{12} J_{T i^{2+}} / J_{T i^{+}}$is strongly affected by the plasma density in front of the target, which is in turn directly proportional to the maximum target current density $j_{\max } \cdot{ }^{13}$ For example, while sputtering Ti in pure $\mathrm{Ar}, J_{T i^{2+}} / J_{T i^{+}}$can be varied from 0.02 to 0.17 by increasing $j_{\max }$ from 0.06 to $1.1 \mathrm{~A} / \mathrm{cm}^{2}$. This is accomplished by increasing the energy $E_{p}$ per HIPIMS pulse from 1 to $10 \mathrm{~J}$ at a constant pulse length of $200 \mu \mathrm{s}$. At the low end of the HIPIMS range, $j_{\max }=0.06 \mathrm{~A} / \mathrm{cm}^{2}$, a current density comparable to conventional dc magnetron sputtering which is known to have a lower plasma density $\left(n_{e} \sim 10^{10} \mathrm{~cm}^{-3}\right.$ [ref.14] compared to $n_{e} \sim 10^{12}-10^{13}$ $\mathrm{cm}^{-3}$ for HIPIMS [ref.15]), and hence a low probability of sputter-ejected metal atom ionization (the mean ionization distance is inversely proportional to $n_{e}$ ). ${ }^{14}$ As a consequence, lowering $J_{T i^{2+}} / J_{T i^{+}}$by reducing $j_{\max }$ has the effect of decreasing the overall degree of metal ionization.

Figure 4 compares the evolution of singly-ionized metal-ion flux $J_{\mathrm{Ti}^{+}}$as a function of $J_{T i^{2+}} / J_{T i^{+}}$for two different types of experiments. In the lower curve, $E_{p}$ (and thus $j_{\max }$ ) is varied from 1 to $10 \mathrm{~J}$ in pure Ar discharges (filled squares), while in the upper curve $\chi_{X e}$ is varied from 0 
to 1 at a constant $E_{p}$ value of $10 \mathrm{~J}$ (open circles). For the former case, decreasing $\mathrm{Ti}^{2+}$ comes at the steep price of a corresponding reduction in the singly-ionized metal-ion flux $J_{T i^{+}}$, from $2.6 \times 10^{8}$ cps with $E_{p}=10 \mathrm{~J}\left(j_{\max }=1.1 \mathrm{~A} / \mathrm{cm}^{2}, J_{T i^{2+}} / J_{T i^{+}}=0.17\right)$ to $5.7 \times 10^{6} \operatorname{cps}$ with $E_{p}=1 \mathrm{~J}\left(j_{\max }=0.06\right.$ $\left.\mathrm{A} / \mathrm{cm}^{2}, J_{T i^{2+}} / J_{T i^{+}}=0.02\right)$, i.e., a decrease by a factor of 45 . This indicates a dramatic reduction in the overall degree of metal ionization, as the total metal flux $J_{T i}$ to the substrate, estimated based upon film thickness obtained from cross-sectional scanning electron microscopy (SEM) images of corresponding Ti layers, decreases by only a factor of two. As a consequence, deposition conditions that correspond to $J_{T i^{2+}} / J_{T i^{+}}=0.02$ resemble those of conventional dc magnetron sputtering for which metal ionization is negligible. In distinct contrast, varying $J_{T i^{2+}} / J_{T i^{+}}$by tuning the gas composition while maintaining HIPIMS pulse parameters constant provides the ability to significantly decrease $J_{T i^{2+}}$ with relatively little effect on $J_{T i^{+}} \cdot J_{T i^{2+}} / J_{T i^{+}}$decreases from 0.17 with $\chi \times e=0$ to 0.01 with $\chi X e=1$, while $J_{T i^{+}}$decreases by a factor of only $2.2 \times$, from $2.6 \times 10^{8}$ to $1.2 \times 10^{8}$ cps.

In Figure 5, the metal-ion flux ratio $J_{T i^{2+}} / J_{T i^{+}}$incident at the substrate position is plotted as a function of the first ionization potential $I P_{g}^{1}$ for the four noble gases. $J_{T i^{2+}} / J_{T i^{+}}$increases with increasing $I P_{g}^{1}$, from 0.01 with $\mathrm{Xe}\left(I P_{X e}^{1}=12.16 \mathrm{eV}\right)$ to 0.03 for $\mathrm{Kr}\left(I P_{K r}^{1}=14.04 \mathrm{eV}\right), 0.17$ for $\mathrm{Ar}$ $\left(I P_{A r}^{1}=15.76 \mathrm{eV}\right)$, and 0.62 for $\mathrm{Ne}\left(I P_{N e}^{1}=21.63 \mathrm{eV}\right)$.

\section{Discussion}

The choice of the noble gas used during sputtering of metal targets in HIPIMS mode has a profound effect on the discharge characteristics as well as on the energy distribution and average charge state of metal ions incident at the substrate and growing film. We show, using Ti as a model target material, that the metal-ion flux ratio $J_{T i^{2+}} / J_{T i^{+}}$at the substrate can be continuously and 
controllably adjusted over a wide range, $0.01 \leq J_{T i^{2+}} / J_{T i^{+}} \leq 0.62$. This provides increased capability for controlling film microstructure, and hence physical properties, while minimizing ionbombardment-induced compressive stress (such as obtained from rare-gas ion irradiation) during HIPIMS film growth utilizing a substrate bias synchronized to the metal-ion dominated portion of the pulses..$^{2,3,5}$ Metal, as opposed to the noble-gas, ions primarily end on lattice sites. ${ }^{5}$

HIPIMS discharges are complex, time-dependent, and operate far from equilibrium. In order to qualitatively describe the general trends observed in these experiments, we use time-averaged parameters (e.g., electron temperature, discharge voltage, and sputter-ejected atom thermalization distance) corresponding to steady-state plasmas.

In low-pressure, cold-cathode, steady-state discharges, each electron must produce, on average, a sufficient number of ions impacting the target that one new secondary electron is emitted. This requires a discharge voltage: ${ }^{16}$

$$
V_{d i s}=E_{g} / \gamma_{g} \mathcal{E}_{i} \mathcal{E}_{\ell},
$$

in which $E_{g}$ is the average electron energy needed to produce an ion by electron-impact ionization, $\gamma_{g}$ is the ion-impact secondary-electron emission coefficient at the target, $\mathcal{E}_{i}$ is the ion collection efficiency, and $\varepsilon_{e}$ is the average fraction of the primary electron energy consumed in ionizing collisions. The product $\mathcal{E}_{i} \varepsilon_{e}$ is close to unity in magnetrons; $;{ }^{16}$ thus, $V_{d i s}$ is primarily controlled by the ratio $E_{g} / \gamma_{g}$.

Observed variations in $j_{\max }$ as a function of gas composition (see Fig. 1) arise from the fact that, with constant pulse energy and length, the average current density is inversely proportional to the average discharge voltage $V_{\text {dis. }} j_{\max }$ values for pure $\mathrm{Ne}$, Ar, and $\mathrm{Ne} / \mathrm{Ar}$ gas mixtures are approximately the same as shown in Fig. 1(a). The Ti secondary-electron emission coefficient $\gamma_{g}$ is larger for $\mathrm{Ne} ;{ }^{17}$ however, because of the higher $\mathrm{Ne}$ ionization potential, the average energy required 
to create an ion is also larger. Thus, the ratio $E_{g} / \gamma_{g}$, and therefore $j_{\max }$, remains approximately constant as a function of $\chi_{g}$ in Ne/Ar mixtures. In $\mathrm{Kr} / \mathrm{Ar}$ and Xe/Ar mixtures, $j_{\text {max }}$ decreases with increasing $\chi_{g}$ (i.e., decreasing Ar concentration) due primarily to the correspondingly lower $\gamma_{g}$ values. ${ }^{17}$

$\mathrm{The} \mathrm{Ti}^{+}$and $\mathrm{Ti}^{2+}$ IEDFs are controlled by the interplay between ionization and mass transport through the gas phase. Changing the noble gas, hence the ionization potential $I P_{g}^{1}$, and the electron impact ionization cross-section $\sigma_{i}$, affects the discharge and plasma parameters, while the mass $m_{g}$ and the gas scattering cross-section of the noble gas species control metal mean free path $\lambda$ and thermalization distance in the gas phase. $I P_{g}^{1}, \sigma_{i}, m_{g}$, and $\lambda$ values for $\mathrm{Ne}, \mathrm{Ar}, \mathrm{Kr}$, and $\mathrm{Xe}$ are listed in Table 1 (note that $\sigma_{i}$ corresponds to maximum values as a function of electron energy). Together, these parameters determine the shape of the $\mathrm{Ti}^{+}$and $\mathrm{Ti}^{2+}$ IEDFs shown in Fig. 2, and account for the large differences observed between $I_{T i^{n+}}\left(E_{i}\right)$ curves for Ne and those obtained for the other noble gases.

IEDFs shown in Fig. 2 are plots of metal-ion energy distributions during 200- $\mu$ s-long HIPIMS pulses. In the early stages of a pulse $(\tau<100 \mu \mathrm{s})$, metal-ion IEDFs resemble very broad Sigmund-Thompson sputtered-species energy distributions; ${ }^{18,19}$ that is, $I_{T i^{n+}}\left(E_{i}\right) \propto E_{i} /\left(E_{i}+\right.$ $\left.E_{b}\right)^{3}$ (in which $E_{b}$ denotes the surface-atom binding energy). ${ }^{18}$ Thus, the original energy distribution of sputter-ejected atoms is preserved since these species undergo few or no collisions due to strong plasma rarefaction. ${ }^{12}$ During the later phase of HIPIMS pulses ( $\left.\tau>150 \mu \mathrm{s}\right), I_{T i^{+}}\left(E_{i}\right)$ and $I_{T i^{2+}}\left(E_{i}\right)$ IEDFs collapse to narrow, low-energy peaks as a result of thermalization, in which the sputtered species lose energy via inelastic collisions with noble-gas atoms, as the target current density and, hence, rarefaction decreases. 
The thermalization distance $\lambda_{t h}$ for sputtered species ejected normal to the target - - i.e., the distance at which their initial velocity $v_{o}$ is reduced to the thermal velocity $v_{t h}--$ is estimated as ${ }^{20}$

$$
\lambda_{t h}=\lambda \eta=\lambda \ln \left(v_{t h} / v_{o}\right) / \ln (\xi)
$$

$\lambda$, a function of ion energy, is the average distance traveled between collisions (the mean free path), $\eta$ is the number of collisions in the ejection direction required to thermalize a sputter-ejected atom, and $\xi$ depends only on the ratio of the sputter-ejected atom mass to that of the sputtering gas. Estimates of $\lambda_{t h}$ based on Eq. (2) should be treated as lower limits since gas rarefaction effects, which are strong in HIPIMS due to high instantaneous sputter-ejected fluxes, further extend the thermalization distance, especially during metal-ion rich portion of the pulse. Estimated $\eta, \lambda$, and $\lambda_{t h}$ values for $\mathrm{Ti}$ atoms passing through $\mathrm{Ne}, \mathrm{Ar}, \mathrm{Kr}$, and Xe discharges at $3 \mathrm{mTorr}$ with an average sputter-ejection energy of $5 \mathrm{eV}$ are listed in Table 1.

Based upon the $\eta$ values given in Table 1, the number of collisions necessary to thermalize sputtered $\mathrm{Ti}$ atoms increases, as expected, with decreasing noble-gas atomic number. $\lambda_{t h}$ is significantly greater than the target/substrate separation only for Ne. This explains the higher average energy, and the lack of low-energy thermalization peaks, in $\mathrm{Ti}^{+}$and $\mathrm{Ti}^{2+}$ IEDFs obtained from Ne HIPIMS discharges (see Fig. 2).

Since electron impact is the primary ionization mechanism in HIPIMS plasmas, ${ }^{12}$ the ionization rate is a strong function of the shape of the electron-energy distribution function $g\left(E_{e}\right)$, which for a steady-state plasma has an approximately Maxwellian form, $g\left(E_{e}\right) \sim \sqrt{E_{e}} e^{-E_{e} / k T_{e}}$, in which $T_{e}$ is the electron temperature and $g\left(E_{e}\right)$ reaches a maximum at $E_{e}=T_{e} / 2$. In plasmas for which $T_{e} \ll I P_{g}^{1}$, the typical case for HIPIMS with $T_{e} \sim 3-5 \mathrm{eV},{ }^{15}$ only electrons in the high-energy tail of the $g\left(E_{e}\right)$ function contribute to the ionization rate. The Thomson cross-section for electronimpact ionization is zero for $E_{e} \ll I P_{g}^{1}$, while for $E_{e}>I P_{g}^{1}$, it varies as $\left(E_{e}-I P_{g}^{1}\right) / I P_{g}^{1} \cdot{ }^{21}$ Thus, 
$g\left(E_{e}\right)$ is expected to strongly deviate from a Maxwellian distribution for $E_{e}>I P_{g}^{1}$. Since $I P_{g}^{1}$ in noble-gas plasmas increases with increasing atomic number, ${ }^{22}$ the choice of sputtering gas determines the shape of the high-energy $g\left(E_{e}\right)$ tail and, thus, the probability for creating multiplyionized metal ions since $I P_{M e}^{2}$ is often comparable to $I P_{g}^{1}$.

Results presented in Figures 2-4 demonstrate that the sputtering-gas composition can be used to tune the average charge state of metal ions incident at the film surface during HIPIMS deposition. For example, $J_{T i^{2+}}$ is reduced by a factor of 37.5 , while $J_{T i^{+}}$drops by only $2.2 \times$ upon replacing Ar with Xe; no multiply-charged $\mathrm{Ti}^{n+}$ ions with $n>2$ are observed. As a consequence, $J_{T i^{2+}} / J_{T i^{+}}$decreases from 0.17 to 0.01 . Such a large effect can be explained by comparing the first and second ionization energies $I P_{M e}^{1}$ and $I P_{M e}^{2}$ of sputtered species to the first ionization energy of the sputtering gas $I P_{g}^{1}$. The production of doubly-ionized metal ions requires electrons with energy $E_{e}>I P_{M e}^{2}$; thus, the creation rate of doubly-ionized species strongly depends on whether $I P_{g}^{1}$ is larger or smaller than $I P_{M e}^{2}$.

If $I P_{M e}^{2}<I P_{g}^{1}$, as is the case for Ti sputtered in $\operatorname{Ar}\left(I P_{T i}^{2}=13.62 \mathrm{eV}\right.$ and $\left.I P_{A r}^{1}=15.76 \mathrm{eV}\right)$, there is a significant electron population in the discharge with energies in the range $I P_{T i}^{2}<E_{e}<$ $I P_{A r}^{1}$, i.e., too low to ionize Ar, yet high enough to produce $\mathrm{Ti}^{2+}$. The situation changes dramatically if $I P_{M e}^{2}>I P_{g}^{1}$, as for Ti sputtered in $\mathrm{Xe}\left(I P_{X e}^{1}=12.16 \mathrm{eV}\right)$. Here, Xe ionization depletes the population of electrons with $E_{e}>I P_{X e}^{1}$ and the $\mathrm{Ti}^{2+}$ creation rate decreases, as shown in Figs. 2-4. Since both $I P_{X e}^{1}$ and $I P_{A r}^{1}$ are significantly higher than $I P_{M e}^{1}\left(I P_{T i}^{1}=6.85 \mathrm{eV}\right)$, the impact on $\mathrm{Ti}^{+}$ production is small. Consequently, varying $\chi_{X e}$ has a large effect on the integrated $J_{T i^{2+}}$ flux incident at the film growth surface, but affects $J_{T i^{+}}$to a far smaller extent. Increasing the difference between $I P_{M e}^{2}$ and $I P_{g}^{1}$, as for Ti sputtered in $\mathrm{Ne}\left(I P_{N e}^{1}=21.63 \mathrm{eV}\right)$, increases the population of 
electrons with energy in the range $I P_{T i}^{2}<E_{e}<I P_{N e}^{1}$, which leads to an increased production of $\mathrm{Ti}^{2+}$ as observed experimentally $\left(J_{T i^{2+}} / J_{T i^{+}}=0.62\right)$.

The ionization potentials of selected metallic elements with atomic numbers from 13 to 79 , including groups IV, V, and VI transition metals (TM) which are relevant for HIPIMS growth of TM nitrides, carbides, and oxides, are shown in Figure 6. The horizontal lines correspond to noblegas $I P_{g}^{1}$ values. In general, both $I P_{M e}^{1}$ and $I P_{M e}^{2}$ increase with decreasing atomic radius, moving from left to right across the periodic table, due to a stronger Coulomb interaction between the nucleus and outer-shell electrons. $I P_{M e}^{1}$ and $I P_{M e}^{2}$ decrease from top to bottom within columns in the periodic table, because of enhanced screening of the nuclear charge by increasing numbers of inner-shell electrons.

The average sputtered metal-ion charge state can be controllably tuned during deposition of a wide range of transition metals for which the conditions $I P_{M e}^{1}<I P_{X e}^{1}$ and $I P_{X e}^{1}<I P_{M e}^{2}<I P_{N e}^{1}$ are satisfied by adjusting mixed noble-gas compositions. Note that extending the upper limit of $I P_{g}^{1}$ to $I P_{H e}^{1}(24.59 \mathrm{eV})^{22}$ is not practical due to extremely low He sputter yields. ${ }^{23}$ Comparison with data in Figure 6 indicates that the approach described here is broadly applicable.

\section{Conclusions}

We demonstrate a strategy for tuning the fraction of multiply-ionized metal ions incident at the growing film surface during HIPIMS deposition. This has been shown, for example, to be essential for controlling phase composition, while minimizing compressive residual stresses, in pseudobinary TM nitride thin films. ${ }^{2,3,8}$ The approach is based on controllably adjusting plasma electron-energy distributions $g\left(E_{e}\right)$ by the proper choice of sputtering-gas mixtures, since the ionization probability of sputtered metal atoms during HIPIMS is determined by the $g\left(E_{e}\right)$ high- 
energy tail. The key parameters are the second ionization potential $I P_{M e}^{2}$ of sputter-ejected metal atoms and the first ionization potential $I P_{g}^{1}$ of the sputtering gas. Using $\mathrm{Ti}$ as a model target material sputtered in the noble gases $\mathrm{Ar}, \mathrm{Ne}, \mathrm{Kr}, \mathrm{Xe}$, and gas mixtures, we employ energy- and timedependent mass spectrometry to measure the $\mathrm{Ti}^{2+} / \mathrm{Ti}^{+}$flux ratio $J_{T i^{2+}} / J_{T i^{+}}$incident at the substrate during HIPIMS (there is no measurable $\mathrm{Ti}^{n+}$ flux with $n>2$ ). We show that $J_{T i^{2+}} / J_{T i^{+}}$can be tuned over a wide range, from 0.01 to 0.62 , for which $J_{T i^{2+}}$ varies strongly while $J_{T i^{+}}$remains approximately constant. This strategy should be applicable to a wide range of metal targets for which the criteria $I P_{X e}^{1}<I P_{M e}^{2}<I P_{N e}^{1}$ is satisfied.

\section{Acknowledgements}

Financial support from the European Research Council (ERC) Advanced Grant \#227754, the VINN Excellence Center Functional Nanoscale Materials (FunMat) Grant \#2005-02666, the Knut and Alice Wallenberg Foundation Grant \#2011.0143 and the Swedish Government Strategic Faculty Grant In Materials Science to Linköping University (Grant SFO Mat-LiU AFM) are gratefully acknowledged. 


\section{Figure captions}

Fig. 1. Target current density $j_{T}$ vs. time $\tau$ waveforms recorded as a function of noble-gas composition during HIPIMS sputtering of $\mathrm{Ti}$ in (a) Ne/Ar, (b) $\mathrm{Kr} / \mathrm{Ar}$, and (c) $\mathrm{Xe} / \mathrm{Ar}$ mixtures. The average target power $\mathcal{P}$ and total gas pressure $P$ were maintained constant at $1 \mathrm{~kW}$ and 0.4 $\mathrm{Pa}$ (3 mTorr), while gas flow rates $f_{g} /\left(f_{g}+f_{A r}\right)$, in which $g=\mathrm{Ne}, \mathrm{Kr}$, or Xe, are varied from 0 to 1 .

Fig. 2. $\mathrm{Ti}^{+}$and $\mathrm{Ti}^{2}$ ion energy distribution functions recorded during HIPIMS pulses while sputtering $\mathrm{Ti}$ in $\mathrm{Ne}, \mathrm{Ar}, \mathrm{Kr}$, and $\mathrm{Xe}$. The average target power $\mathcal{P}$ and total gas pressure $P$ were maintained constant at $1 \mathrm{~kW}$ and $0.4 \mathrm{~Pa}(3 \mathrm{mTorr})$ respectively.

Fig. 3. The $\mathrm{Ti}^{2+} / \mathrm{Ti}^{+}$integrated flux ratio $J_{T i^{2+}} / J_{T i^{+}}$at the substrate position, plotted as a function of noble-gas composition for Ne/Ar, $\mathrm{Kr} / \mathrm{Ar}$, and Xe/Ar mixtures during HIPIMS sputtering of a Ti target. The average target power $\mathcal{P}$ and total gas pressure $P$ were maintained constant at $1 \mathrm{~kW}$ and $0.4 \mathrm{~Pa}$ (3 mTorr), while gas flow rates $f_{g} /\left(f_{g}+f_{A r}\right)$ are varied from 0 to 1.

Fig. 4. Singly-charged $\mathrm{Ti}^{+}$ion flux $J_{T i^{+}}$plotted as a function of the $\mathrm{Ti}^{2+} / \mathrm{Ti}^{+}$flux ratio $J_{T i^{2+}} / J_{T i^{+}}$ incident at the substrate position during HIPIMS sputtering of a Ti target. Two different types of experiments are carried out: the target peak current density $j_{\max }$ is varied while sputtering in pure $\operatorname{Ar}$ (filled squares), and the composition $\chi_{X e}$ of the Xe/Ar sputtering gas mixture is varied while maintaining HIPIMS parameters constant (open circles). In the first set of experiments, $j_{\max }$ is adjusted by varying the $\operatorname{target}$ power $\mathcal{P}$ from 0.1 to $1 \mathrm{~kW}$ with the 
gas pressure $P$ maintained constant at $0.4 \mathrm{~Pa}(3 \mathrm{mTorr})$. In the second set, $\chi_{x e}$ is varied from 0 to 1 at a constant target power $\mathcal{P}=1 \mathrm{~kW}$ and pressure $P=0.4 \mathrm{~Pa}$ (3 mTorr).

Fig. 5. The $\mathrm{Ti}^{2+} / \mathrm{Ti}^{+}$integrated flux ratio $J_{T i^{2+}} / J_{T i^{+}}$, measured at the substrate position during HIPIMS sputtering of a Ti target, plotted as a function of the first ionization potential $I P_{g}^{1}$ of the sputtering gas ( $\mathrm{Ne}, \mathrm{Ar}, \mathrm{Kr}$, or $\mathrm{Xe})$. The average target power $\mathcal{P}$ and total gas pressure $P$ were maintained constant at $1 \mathrm{~kW}$ and $0.4 \mathrm{~Pa}(3 \mathrm{mTorr})$.

Fig. 6. The ionization potentials $I P$ of selected solid elements with atomic numbers from 13 to 79 ; the filled squares and circles correspond to first and second IP values, respectively. The horizontal lines indicate first ionization potentials $I P_{g}^{1}$ for the noble gases $\mathrm{Ne}, \mathrm{Ar}, \mathrm{Kr}$, and Xe.

Table 1. Parameters relevant for HIPIMS sputtering of a Ti target in $\mathrm{Ne}, \mathrm{Ar}, \mathrm{Kr}$, and $\mathrm{Xe}$. Noble-gas mass $m_{g}$, atomic radii $r_{g},{ }^{20}$ first ionization potential $I P_{g}^{1},{ }^{22}$ and maximum electron-impact ionization cross-section $\sigma_{i},{ }^{10}$ the $\mathrm{Ti}$ ion-impact secondary-electron emission coefficient $\gamma_{Y i},{ }^{17}$ the number of collisions $\eta$ required to thermalize sputtered Ti atoms ejected with 5 $\mathrm{eV}$ in the forward direction (see Eq. (2)); the average sputtered Ti atom mean free path $\lambda ;{ }^{20}$ and the thermalization distance $\lambda_{t h}$ (Eq. (2)) for sputtered Ti atoms ejected with $5 \mathrm{eV}$ in the forward direction. 


\section{References}

${ }^{1}$ V. Kouznetsov, K. Macak, J.M. Schneider, U. Helmersson and I. Petrov, Surf. Coat. Technol. 122 (1999) 290

${ }^{2}$ G. Greczynski, J. Lu, M. Johansson, J. Jensen, I. Petrov, J.E. Greene, and L. Hultman, Surf. Coat. Technol. 206 (2012) 4202

${ }^{3}$ G. Greczynski, J. Lu, M. Johansson, J. Jensen, I. Petrov, J.E. Greene, and L. Hultman, Vacuum 86 (2012) 1036

${ }^{4}$ I. Petrov, P.B. Barna, L. Hultman, and J.E. Greene, J. Vac. Sci. Technol. A 21 (2003) S117

${ }^{5}$ G. Greczynski, J. Lu, J. Jensen, I. Petrov, J.E. Greene, S. Bolz, W. Kölker, Ch. Schiffers, O. Lemmer and L. Hultman, J. Vac. Sci. Technol. A 30 (2012) 061504

${ }^{6}$ G. Greczynski, J. Jensen, J. Böhlmark, and L. Hultman, Surf. Coat. Technol. 205 (2010) 118

${ }^{7}$ G. Greczynski, J. Jensen, L. Hultman, IEEE Transactions on Plasma Science 38 (2010) 3046

${ }^{8}$ G. Greczynski, J. Lu, J. Jensen, I. Petrov, J.E. Greene, S. Bolz, W. Kölker, Ch. Schiffers, O.

Lemmer and L. Hultman, Thin Solid Films 556 (2014) 87

${ }^{9}$ G. Greczynski and L. Hultman, Vacuum 84 (2010) 1159-1170

${ }^{10}$ R. Rejoub, B. G. Lindsay, and R. F. Stebbings, Phys. Rev. A 65, 042713 (2002)

${ }^{11}$ I. Petrov, I. Ivanov, V. Orlinov, J.-E. Sundgren, J. Vac. Sci. Technol. A, 112733 (1993)

12 J.T. Gudmundsson, N. Brenning, D. Lundin, and U. Helmersson, J. Vac. Sci.

Technol. A, 30030801 (2012)

${ }^{13}$ G. Greczynski, J. Jensen, L. Hultman, Thin Solid Films 519 (2011) 6354-6361

${ }^{14}$ See for instance: see e.g., J.A.Hopwood Plasma Physics in Ionized Physical vapor Deposition, Ed. by J.A.Hopwood, Adademic Press

${ }^{15}$ A. Anders, Surf. Coat. Technol. 257 (2014) 308

16 J.A. Thornton, A.S. Penfold in J.L. Vossen, W. Kern (Eds.), Thin Film Processes, Academic

Press, New York (1978), p. 75

${ }^{17} \gamma_{\mathrm{Ti}}=0.032 \times\left(0.78 \times I P_{g}^{1}-2 \times \mathrm{WF}\right)$, where $I P_{g}^{1}$ is the noble-gas ionization potential and WF is the target work function. See: R.A. Baragiola, E.V. Alonso, J. Ferron, and A. Oliva-Florio, Surf. Sci. 90, 240 (1970).

${ }^{18}$ P. Sigmund, J. Vac. Sci. Technol. A 17, 396-399 (1979)

${ }^{19}$ M. W. Thompson, Physics Reports 69, 335-371 (1981)

${ }^{20}$ W.D. Westwood, J. Vac. Sci. Technol. 15 (1978) 1

${ }^{21}$ M.A. Lieberman, A.J. Lichtenberg "Principles of plasma discharges and materials processing", J.Wiley \& Sons, Inc. New York, 1994, Chapter 3

${ }^{22}$ David R. Lide (ed), CRC Handbook of Chemistry and Physics, 84th Edition. CRC Press. Boca Raton, Florida, 2003; Section 10, Atomic, Molecular, and Optical Physics; Ionization Potentials of Atoms and Atomic Ions

23 N. Matsunami, Y. Yamamura, Y. Itikawa, N. Itoh, Y. Kazumata, S. Miyagawa, K. Morita, R. Shimizu, and H. Tawara, "Energy Dependence of the Yields of Ion-Induced Sputtering of Monatomic Solids", (Inst. Plasma Physics, Nagoya Univ., Japan, 1983) 

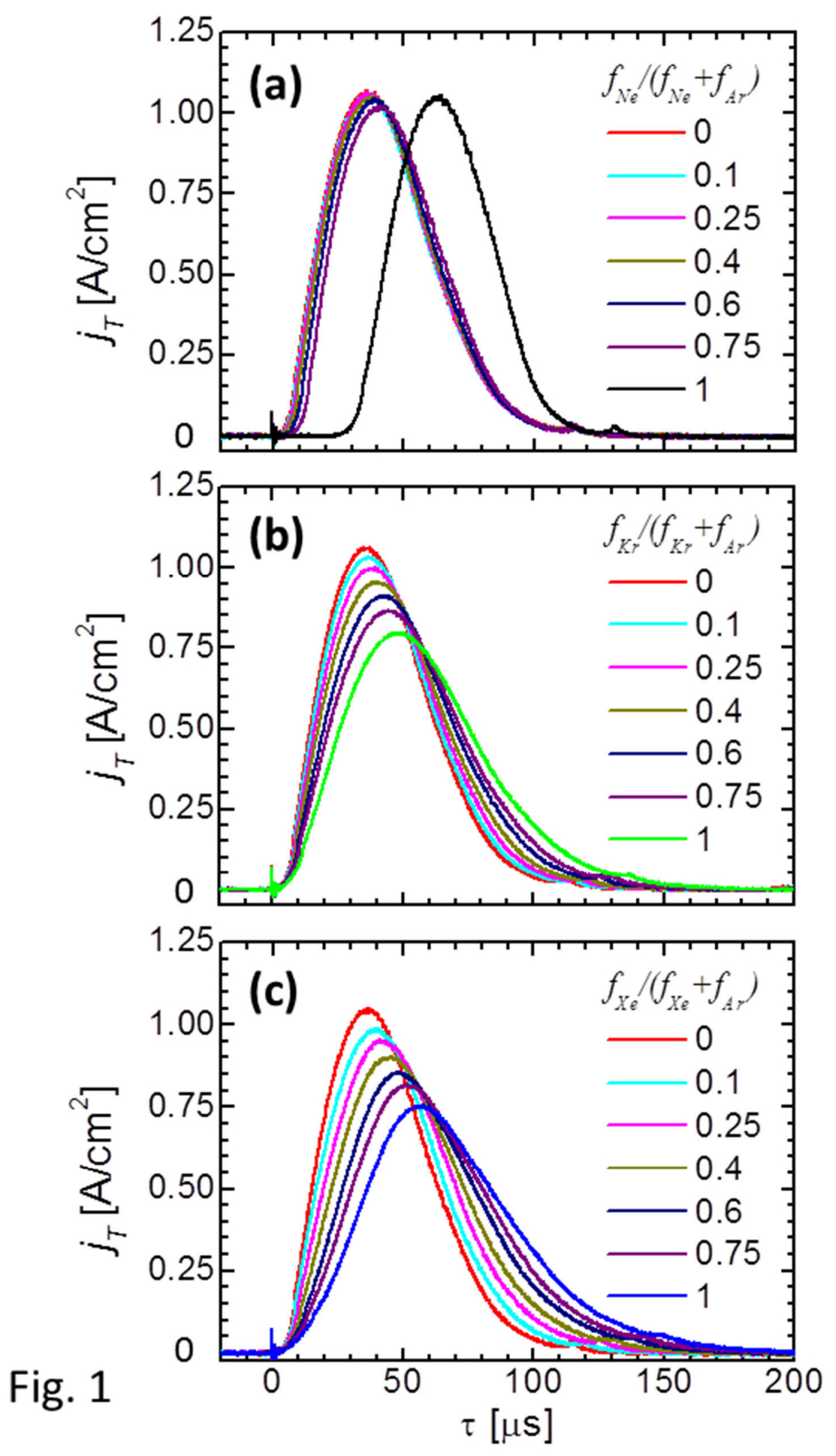

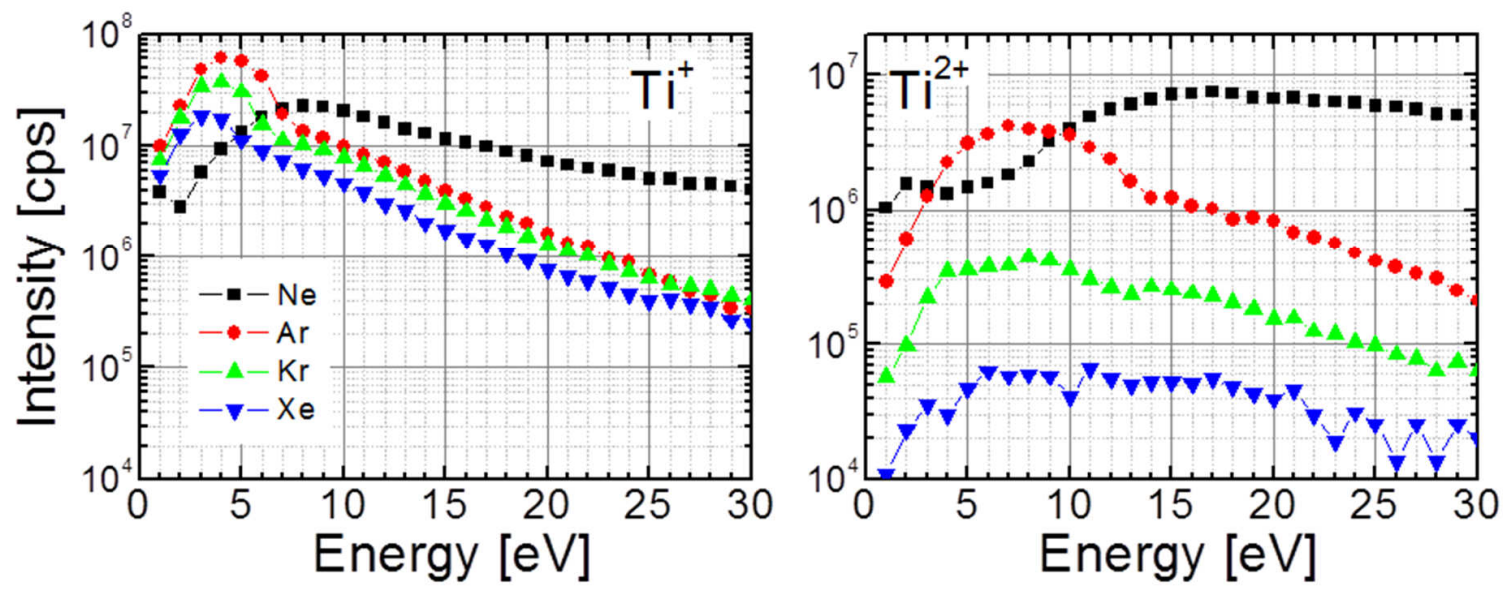

Fig. 2 


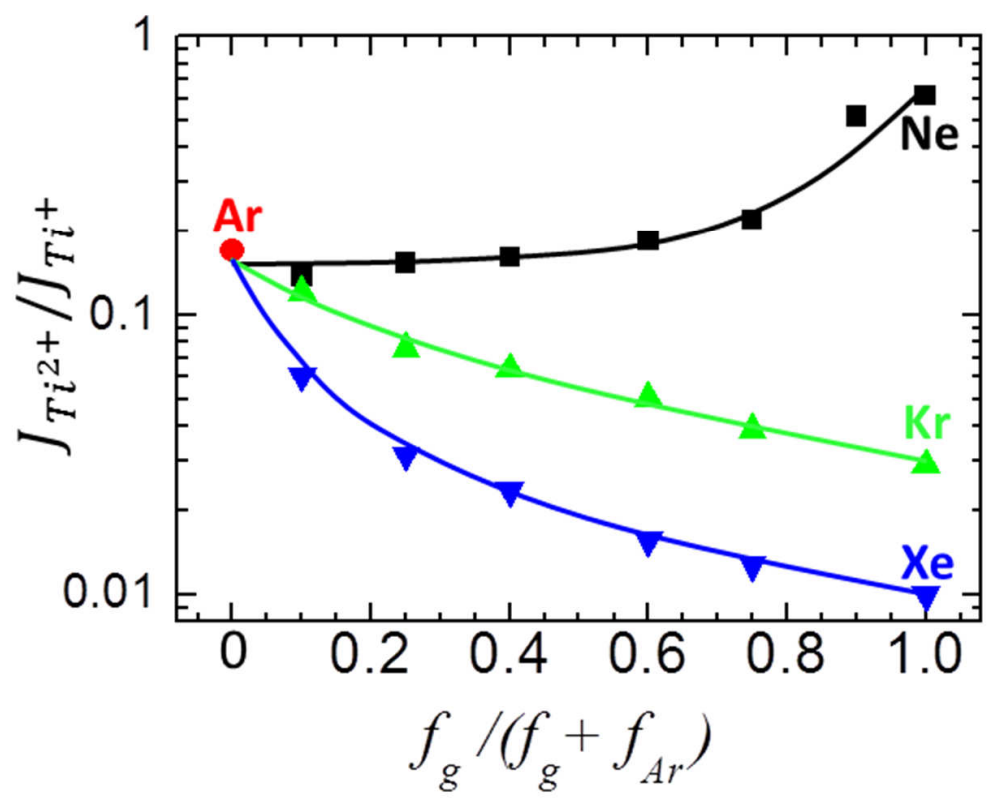

Fig. 3 


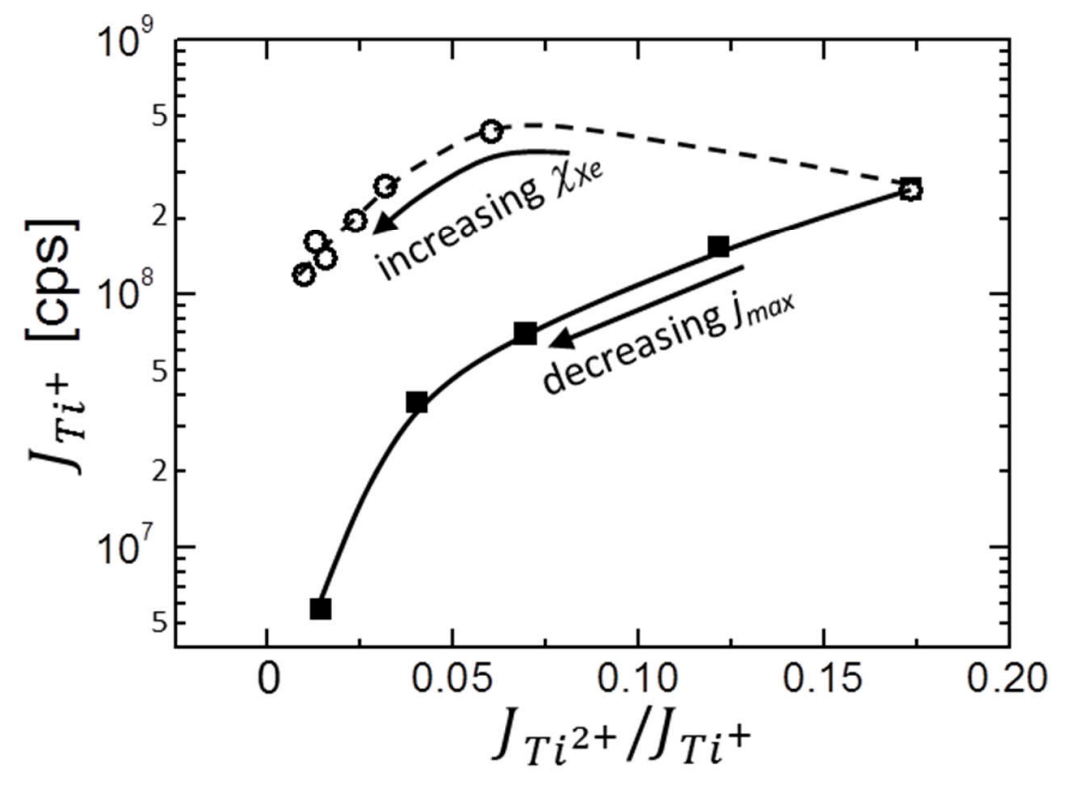

Fig. 4 


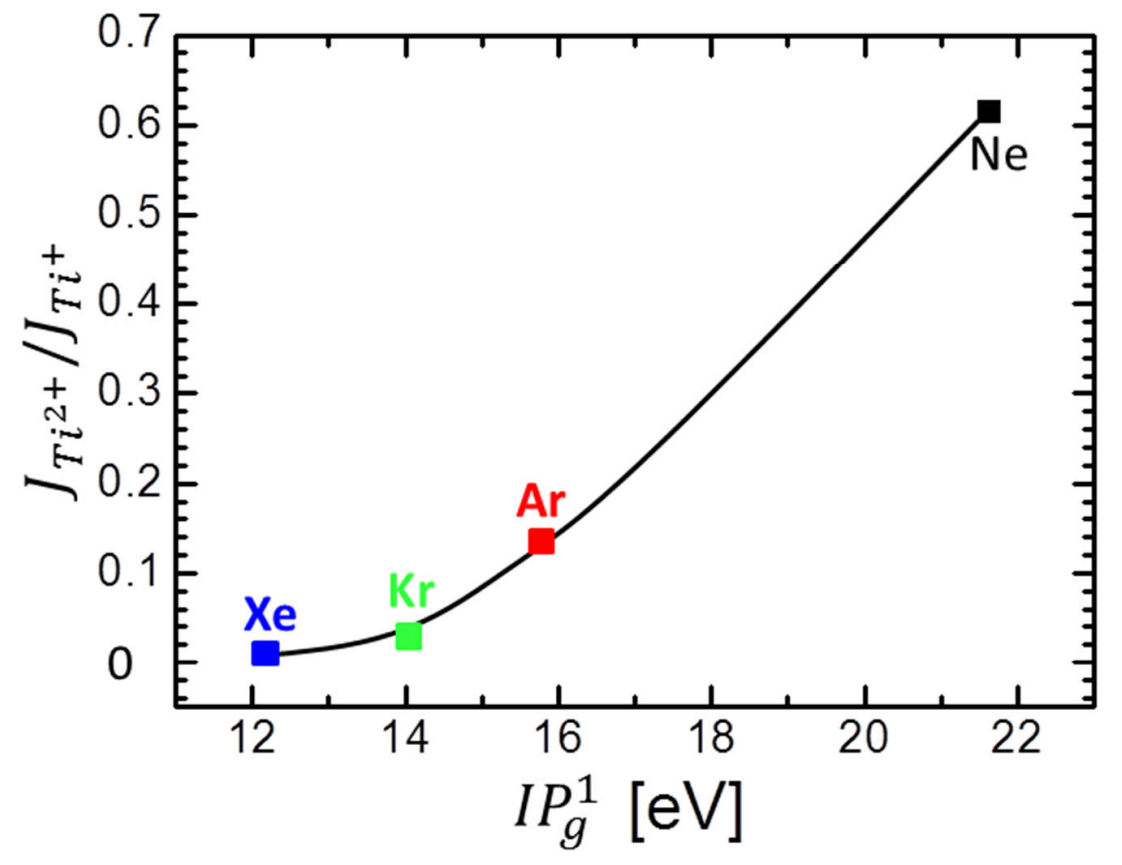

Fig. 5 


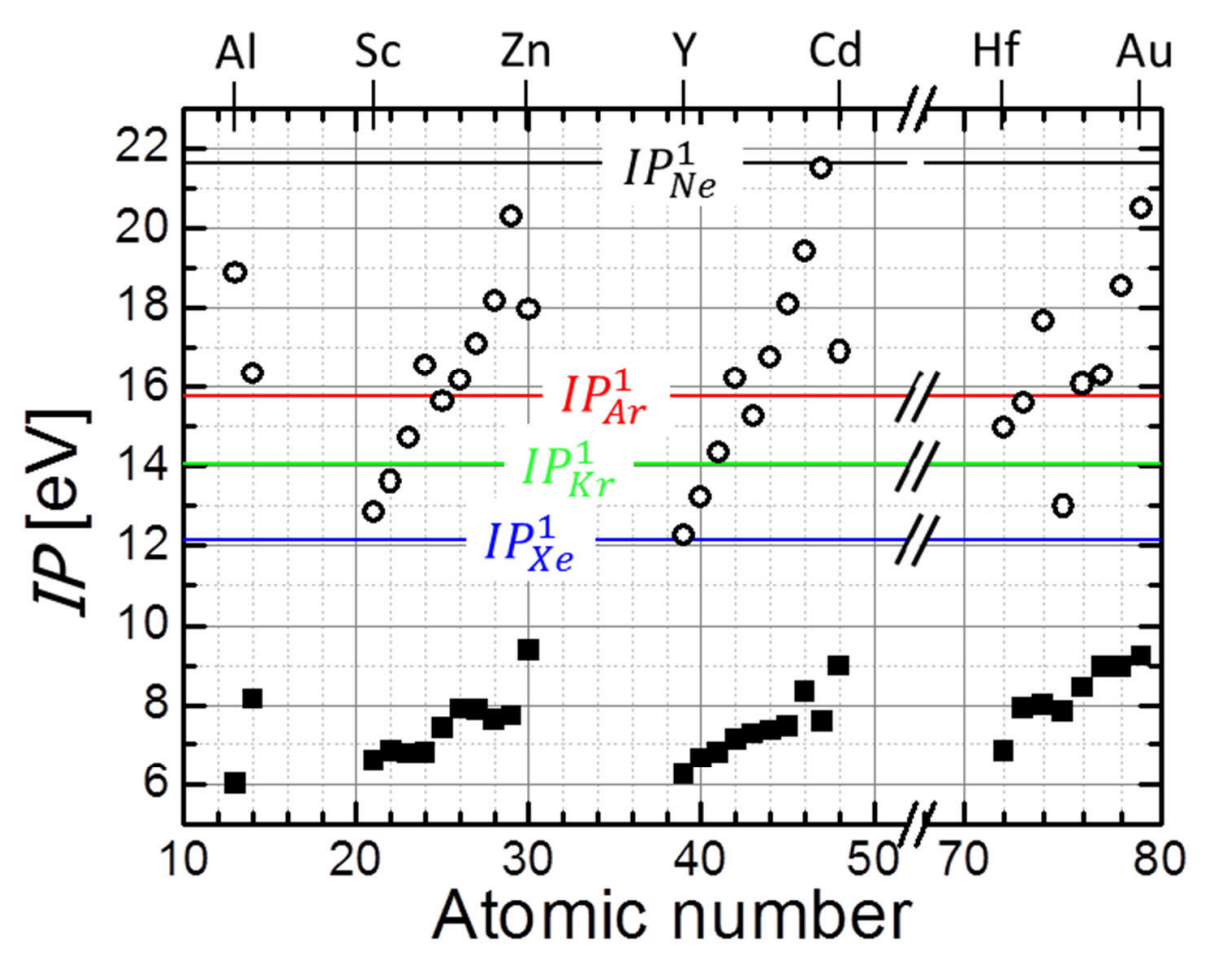

Fig. 6 


\begin{tabular}{|c|c|c|c|c|c|c|c|c|}
\hline $\begin{array}{l}\text { Property } \\
\text { Inert gas }\end{array}$ & $\begin{array}{c}\boldsymbol{m}_{g} \\
\text { [amu] }\end{array}$ & $\begin{array}{c}r_{g} \\
{[\AA ̊]}\end{array}$ & $\begin{array}{l}I P_{g}^{1} \\
{[\mathrm{eV}]}\end{array}$ & $\begin{array}{c}\sigma_{i} \\
{\left[10^{-16} \mathrm{~cm}^{2}\right]}\end{array}$ & $\gamma_{i i}$ & $\eta$ & $\begin{array}{c}\lambda \\
{[\mathrm{cm}]}\end{array}$ & $\begin{array}{r}\lambda_{t h} \\
{[\mathrm{~cm}]}\end{array}$ \\
\hline $\mathrm{Ne}$ & 20.18 & 1.25 & 21.63 & 0.68 & 0.27 & 6.6 & 5.0 & 33.0 \\
\hline $\mathrm{Ar}$ & 39.95 & 1.45 & 15.76 & 2.55 & 0.12 & 3.2 & 4.6 & 14.7 \\
\hline $\mathrm{Kr}$ & 83.30 & 1.75 & 14.04 & 3.49 & 0.07 & 2.2 & 4.7 & 10.3 \\
\hline Xe & 131.29 & 2.00 & 12.16 & 4.67 & 0.03 & 1.8 & 5.1 & 9.2 \\
\hline
\end{tabular}

Table 1 